\title{
Circulating microRNAs indicate cardioprotection by sevoflurane inhalation in patients undergoing off-pump coronary artery bypass surgery
}

\author{
XIAO LIU*, XIAOPENG LIU*, RUIKE WANG, HUI LUO, GANG QIN, \\ LU WANG, ZHI YE, QULIAN GUO and E WANG \\ Department of Anesthesiology, Xiangya Hospital, Central South University, Changsha, Hunan 410008, P.R. China
}

Received January 16, 2015; Accepted March 8, 2016

DOI: 10.3892/etm.2016.3197

\begin{abstract}
In patients undergoing off-pump coronary artery bypass surgery (OPCAB), it is important to attenuate myocardium injury during the surgery. The present study aimed to observe the cardioprotection induced by sevoflurane induction and maintenance compared with propofol intravenous anesthesia, and to detect its potential protection against acute myocardial injury with sensitive biomarkers. In total, 36 patients undergoing $\mathrm{OPCAB}$ were randomly assigned into two groups, receiving sevoflurane $(n=18)$ or propofol $(n=18)$ as the induction and maintenance anesthetic agent. The depth of anesthesia in the two groups was kept at a bispectral index value of 40-50. Physiological and hemodynamic parameters were recorded during the surgery. Cardiac troponin-I (cTnI), creatine kinase-MB (CK-MB), lactate dehydrogenase (LDH) and two microRNAs (miR-499 and miR-208b) were also measured during and subsequent to surgery. Nno statistically significant differences were observed in the physiological and hemodynamic parameters between the two groups prior to surgery. Following surgery, the cardiac output and stroke volume improved significantly in the sevoflurane group $(\mathrm{P}<0.05)$. In addition, patients in the sevoflurane group had lower miR-499 $(\mathrm{P}<0.05)$ and miR-208b $(\mathrm{P}<0.01)$ levels at $12 \mathrm{~h}$ after surgery when compared with the propofol group. However, no significant differences in cTnI, CK-MB and LDH levels were observed following surgery between the two groups. In conclusion, volatile induction and maintenance with sevoflurane resulted in some extent of cardiac function improvement in patients undergoing OPCAB.
\end{abstract}

Correspondence to: Professor E Wang, Department of Anesthesiology, Xiangya Hospital, Central South University, 87 Xiangya Road, Changsha, Hunan 410008, P.R. China

E-mail: ewang324@sina.com

*Contributed equally

Key words: circulating microRNAs, cardioprotection, sevoflurane, off-pump coronary artery bypass surgery
Cardioprotection by sevoflurane is suggested by reduced cardiac injury compared with propofol, and indicated by the sensitive biomarkers, circulating miR-499 and miR-208b.

\section{Introduction}

Coronary artery bypass (CAB) grafting is the main surgical treatment for coronary heart disease, which increases oxygen supply by coronary revascularization. Novel surgical techniques have been developed, such as off-pump CAB (OPCAB) surgery, which is currently widely used. OPCAB has a reduced effect on the physiological circulation (1); however, myocardial ischemia-reperfusion injury (MIRI) remains inevitable. Local and systemic inflammatory responses are considered to serve an important role in MIRI, but its development is also associated with the release of free radicals, calcium overload and ATP reduction. MIRI leads to decreased myocardial contractility, apoptosis of myocytes, subendocardial hemorrhage and myocardial infarction $(2,3)$. Therefore, it is important to mitigate MIRI and protect the myocardium during the $\mathrm{CAB}$ surgery.

Sevoflurane is an inhaled anesthetic frequently used in cardiac surgery, since it has been reported to provide cardiac protection against MIRI by pre- or post-conditioning (4-9). Pre-conditioning by sevoflurane may improve the long-term outcomes of patients, decrease their mortality and morbidity rates, and prolong their survival $(10,11)$. A previous meta-analysis included 22 studies on sevoflurane, involving 1,922 patients undergoing cardiac surgery (12). The study showed that desflurane and sevoflurane have cardioprotective effects that result in decreased morbidity and mortality. It was also shown that the selection of an anesthetic regimen based on halogenated anesthetics was associated with an improved outcome subsequent to cardiac surgery (12). The majority of previous clinical studies have selected to use inhaled anesthetics in combination with intravenous administration of propofol (12).

Cardiac troponins have been introduced as cardiac markers $(13,14)$. Cardiac troponin I (cTnI) determination has been shown to have a potentially high diagnostic efficacy for cardiac ischemic injury (13). In addition, the nonspecific marker lactate dehydrogenase (LDH) and the cardiac-specific 
enzyme creatine kinase-MB (CK-MB) are frequently-used markers of cardiac infarction (15). However, these biomarkers are not consistently sensitive following ischemia onset, requiring repetitive measurements (16).

MicroRNAs (miRNAs) are short, non-coding small RNAs that regulate the expression of proteins by translational repression or degradation of mRNAs (17). In the last decade, studies revealed that miRNAs serve a crucial role in cardiac development and homeostasis, and that their expression is altered in the patients with heart diseases $(18,19)$. It was also demonstrated that circulating miRNAs may be potential novel early biomarkers of cardiac injury and may help improve the management of suspected acute coronary syndrome (ACS) patients (20). In particular, miR-208b and miR-499 levels can provide a reasonable prognosis of left ventricular dysfunction (21).

In the present study, sevoflurane inhalation was used to perform volatile induction and maintenance of anesthesia (VIMA), and its effect was compared with that of total intravenous anesthesia (TIVA) with propofol. In addition, the cardioprotection induced by sevoflurane compared with propofol in off-pump CABG was evaluated using miR-208b and miR-499 as diagnostic biomarkers.

\section{Patients and methods}

Patient selection. In the present single-center randomized controlled study, 36 patients diagnosed with coronary artery disease and scheduled to undergo OPCAB were enrolled between January 2010 and December 2012. The patient characteristics were as follows: Age, 34-70 years; American Society of Anesthesiology physical status classification II-III; New York Heart Association (NYHA) functional classification II-III; and a body-mass index (BMI) of $<30 \mathrm{~kg} / \mathrm{m}^{2}$. The study was approved by the Ethical Committee of Xiangya Hospital, Central South University (Changsha, China). All ongoing and associated trials for this intervention were registered. Written informed consent was obtained from all the patients. The exclusion criteria included emergency surgery, left ventricular ejection fraction of $<40 \%$, diabetes, previous administration of K-ATP channel antagonists or agonists, previous myocardial infarction, history of allergy to anesthetics and abnormal coagulate function. All patients were administered 25-100 mg metoprolol (AstraZeneca plc, London, UK) and 10-20 mg lovastatin (Yangzijiang Pharmaceutical Group, Wuhan, China) per day as preoperative medications. Coronary artery revascularization by OPCAB grafting was performed by one surgeon using two or three coronary vessel grafts per patient and the Octopus 4.3 Tissue Stabilizer (Medtronic, Inc, Minneapolis, MN, USA).

Anesthetic technique. The 36 patients undergoing OPCAB were randomly divided into two groups, namely the sevoflurane and propofol groups ( $\mathrm{n}=18$ each). For the random allocation of patients into the groups, a computer-generated list of random numbers was used. Two additional participants were excluded from each group due to a change to the surgical method. In total, 10 mg morphine (Renfu Pharmaceutical Co., Ltd., Yichang, China) and $0.3 \mathrm{mg}$ scopolamine (Hefeng Pharmaceutical, Co., Ltd., Shanghai, China) were administrated $30 \mathrm{~min}$ before the anesthesia. The electrocardiogram (ECG), blood pressure
(BP), heart rate (HR) and pulse oxygen saturation were continuously monitored using the CARESCAPE Monitor B850 (GE Healthcare, Dallas, TX, USA), and the bispectral index (BIS) value (BIS VISTA ${ }^{\mathrm{TM}}$ monitoring system; Aspect Medical Systems, Inc., Norwood, MA, US) was continuously recorded. Invasive monitoring lines, including a radial artery catheter (Edwards Lifesciences Corp., Irvine, CA, USA), a jugular vein catheter (B. Braun Melsungen AG, Melsungen, Germany) and a Swan-Ganz catheter (Edwards Lifesciences Corp.), were inserted under local anesthesia. Anesthesia was induced with $1-8 \%$ sevoflurane inhalation (Maruishi Pharmaceutical Co., Ltd., Osaka, Japan) in the sevoflurane group, or with 2-4 $\mathrm{mg} / \mathrm{kg}$ propofol injection (AstraZeneca plc) in the propofol group, until the BIS reached 45. Subsequent to administration of $1.0 \mu \mathrm{g} / \mathrm{kg}$ sufentanil (Renfu Pharmaceutical Co., Ltd.) and $0.15 \mathrm{mg} / \mathrm{kg}$ vecuronium (Enhua Pharmaceutical Group Co., Ltd., Xuzhou, China), the patient was intubated and mechanically ventilated, and the ventilator parameters were adjusted to maintain the end-tidal $\mathrm{CO}_{2}$ level at $40-45 \mathrm{mmHg}$. All patients received an intravenous infusion of $10-15 \mathrm{ng} / \mathrm{kg} / \mathrm{min}$ sufentanil and $1.0 \mu \mathrm{g} / \mathrm{kg} / \mathrm{min}$ vecuronium during the surgery. In addition, anesthesia was maintained with a 0.6-1.5 minimum alveolar concentration (MAC) of sevoflurane in the sevoflurane group or $4-8 \mathrm{mg} / \mathrm{kg} / \mathrm{h}$ propofol in the propofol group, in order to maintain a BIS value of 40-50. Patients received routine monitoring for OPCAB using the Vigilance II Monitor (Edwards Lifesciences Corp.) to monitor the mean arterial pressure (MAP), pulmonary artery mean pressure (PAMP), pulmonary artery wedge pressure (PAWP), central vein pressure and core temperature. Nitroglycerin (Yimin Pharmaceutical Co., Ltd., Beijing, China), esmolol (Qilu Pharmaceutical Co., Ltd., Jinan, China) and phenylephrine (Hefeng Pharmaceutical, Co., Ltd.) were administered, according to hemodynamic parameters.

Hemodynamic parameters, including systolic BP, diastolic BP, MAP and PAMP, were monitored automatically and recorded prior to anesthesia, intraoperatively and postoperatively. In addition, the central venous pressure (CVP), cardiac output (CO) and stroke volume (SV) were recorded using pulmonary artery catheters. Furthermore, blood samples were obtained subsequent to anesthesia induction and surgery, and were immediately centrifuged at 3,000 x g for $10 \mathrm{~min}$ at $4^{\circ} \mathrm{C}$. The serum was stored at $-70^{\circ} \mathrm{C}$ for measurement of cTnI, CK-MB, LDH, miR-499 and miR-208b expression levels. Laboratory technicians were blinded to the sample groups.

Determination of quantitative cTnI, $C K-M B$ and $L D H$ cardiac marker expression levels using enzyme-linked immunosorbent assay (ELISA). The details of the series were unknown to any of the investigators who performed the laboratory tests. Circulating cTnI protein concentration was measured by an ELISA. The method was based on a single-step sandwich principle (22), performed according to the instructions of the human cTnI ELISA kit (cat. no. GWB-83A61F; GenWay Biotech Inc., San Diego, CA, US). cTnI measurements were performed in duplicate and recorded by a batch ELISA analyzer (iMark 680 microplate absorbance reader; Bio-Rad Laboratories, Inc., Richmond, CA, US). In addition, the cardiac isoenzymes CK-MB and LDH were detected using commercially available kits (cat. nos. 04525299190 and 11644793001 , respectively; Roche 
Table I. Patient characteristics.

\begin{tabular}{lcc}
\hline Parameter & Sevoflurane group $(\mathrm{n}=18)$ & Propofol group $(\mathrm{n}=18)$ \\
\hline Gender, male/female & $11 / 7$ & $13 / 5$ \\
Age (year) & $63.3 \pm 7.2$ & $61.8 \pm 10.8$ \\
Body mass index $\left(\mathrm{kg} / \mathrm{m}^{2}\right)$ & $26.5 \pm 3.2$ & $25.8 \pm 2.7$ \\
NYHA level, II/III & $7 / 11$ & $8 / 10$ \\
Number of grafts, $2 / 3$ & $8 / 10$ & $8 / 10$ \\
Surgery duration (min) & $247.8 \pm 31.5$ & $253.3 \pm 33.7$ \\
\hline Data are presented as the mean \pm standard deviation or absolute numbers. No statistically significant differences were observed between the & \\
two groups (P>0.05). & & \\
\hline
\end{tabular}

Table II. Intraoperative hemodynamic and cardiac function measurements.

\begin{tabular}{|c|c|c|c|c|c|c|}
\hline \multirow[b]{2}{*}{ Measurement } & \multicolumn{3}{|c|}{ Sevoflurane group } & \multicolumn{3}{|c|}{ Propofol group } \\
\hline & Preoperative & Postoperative & P-value & Preoperative & Postoperative & P-value \\
\hline HR (beats/min) & $71.7 \pm 12.5$ & $77.3 \pm 10.4$ & 0.163 & $69.3 \pm 11.5$ & $75.6 \pm 10.5$ & 0.106 \\
\hline MAP (mmHg) & $95.3 \pm 14.3$ & $84.1 \pm 12.5$ & 0.027 & $92.1 \pm 17.6$ & $78.8 \pm 13.4$ & 0.029 \\
\hline CVP (mmHg) & $11.4 \pm 3.4$ & $12.5 \pm 2.7$ & 0.381 & $10.8 \pm 3.0$ & $12.4 \pm 2.5$ & 0.067 \\
\hline PAMP (mmHg) & $23.0 \pm 5.6$ & $25.0 \pm 5.7$ & 0.288 & $22.8 \pm 5.1$ & $26.2 \pm 6.3$ & 0.092 \\
\hline PAWP (mmHg) & $15.7 \pm 3.7$ & $16.2 \pm 3.4$ & 0.588 & $15.6 \pm 3.1$ & $16.5 \pm 3.9$ & 0.482 \\
\hline $\mathrm{CO}(1 / \mathrm{min})$ & $4.5 \pm 0.9$ & $5.5 \pm 0.7$ & 0.002 & $4.6 \pm 1.1$ & $5.1 \pm 1.1$ & 0.121 \\
\hline $\mathrm{SV}(\mathrm{ml})$ & $62.8 \pm 10.7$ & $72.3 \pm 12.7$ & 0.034 & $66.8 \pm 12.9$ & $69.1 \pm 11.6$ & 0.476 \\
\hline
\end{tabular}

Data are presented as the mean \pm standard deviation. No statistically significant differences were observed between the two groups at the same time points $(\mathrm{P}>0.05)$. HR, heart rate; MAP, mean arterial pressure; CVP, central venous pressure; PAMP, pulmonary arterial mean pressure; PAWP, pulmonary artery wedge pressure; $\mathrm{CO}$, cardiac output; SV, stroke volume.

Diagnostics, Indianapolis, IN, USA) using an autoanalyzer system (Cobas 4000; Roche Diagnostics).

Determination of miR-208b and miR-499 cardiac marker expression levels using reverse transcription-quantitative polymerase chain reaction $(R T-q P C R)$. The serum, which had been stored at $-70^{\circ} \mathrm{C}$ in RNAse-free tubes, was processed for miRNA analysis by RT-qPCR. Total RNA was isolated using TRIzol LS reagent (cat. no. 15596-026; Thermo Fisher Scientific Inc., Carlsbad, CA, USA). Genomic DNA contamination was eliminated using a DNAse I kit (cat. no. 89836; Fermentas; Thermo Fisher Scientific Inc., Vilnius, Lithuania) and RNA concentration was quantified with a spectrophotometer (NanoDrop ND-1000; Thermo Fisher Scientific Inc., Wilmington, DE, USA). The RNA integrity of small RNAs was determined using small RNA Chip analysis kit (cat. no. 5067-1548) along with an Agilent 2100 Bioanalyzer (Agilent Technologies Inc., Santa Clara, CA, US). A total of $2 \mu \mathrm{g}$ DNA-free RNA was reverse transcribed into cDNA using the TaqMan miRNA Reverse Transcription kit (cat. no. 4366596; Thermo Fisher Scientific Inc., Carlsbad, CA, USA). qPCR was performed on a QuantStudio ${ }^{\text {TM }} 7$ Flex Real Time PCR System (Applied Biosystems; Thermo Fisher Scientific, Inc.) using the TaqMan Fast Advanced Master Mix (cat. no. 4444556; Thermo Fisher Scientific,
Inc.) and miRNA-specific primers as follows: miR-208b forward, 5'-CCATAAGACGAACAAAAGGT-3', and reverse, 5'-GTGCAGGGTCCGAGGT-3'; miR-499 forward, 5'-ACA GACTTGCTGTGATG-3', and reverse, 5'-GTGCAGGGT CCGAGGT-3'; and RNU6 forward, 5'-CTCGCTTCG GCAGCACA-3', and reverse, 5'-AACGCTTCACGAATT TGCGT-3' (Applied Biosystems; Thermo Fisher Scientific Inc.). The PCR conditions were as follows: $95^{\circ} \mathrm{C}$ for $15 \mathrm{~min}$, followed by 35 cycles at $94^{\circ} \mathrm{C}$ for $15 \mathrm{sec}, 55^{\circ} \mathrm{C}$ for $30 \mathrm{sec}$ and $72^{\circ} \mathrm{C}$ for $30 \mathrm{sec}$. RT-qPCR amplification was performed in duplicate using 1:5 diluted RT products for miR-208b and miR-499. All RT-qPCR data were normalized to RNU6, and the RNU6 levels did not differ between the two groups. After normalization for RNU6, the relative gene expression was calculated by the $2^{-\Delta \Delta \mathrm{Cq}}$ method (23).

Statistical analysis. All data for variables are expressed as the mean \pm standard deviation. For comparison between the two groups, the Mann-Whitney test was used, while categorical data were analyzed using $\chi^{2}$-test. The hemodynamic and serological data were analyzed using repeated measures analysis of variance (ANOVA), followed by a Bonferroni post-hoc correction or non-parametric test in cases where the ANOVA prerequisite was not met. A P-value of $<0.05$ was considered to indicate statistically significant differences. GraphPad Prism-5 


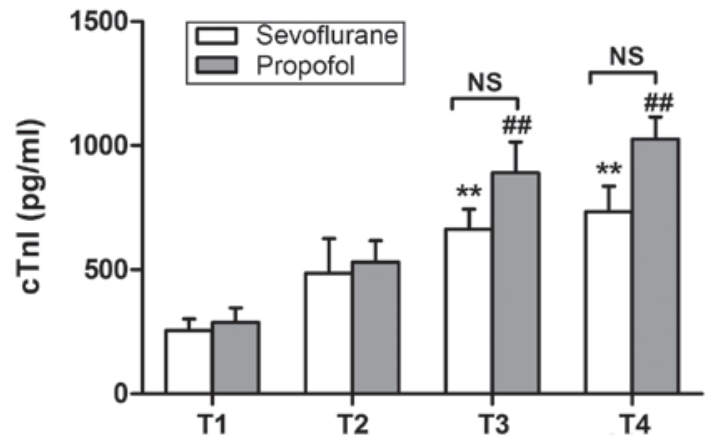

Figure 1. cTnI expression levels preoperatively (T1), and at $6 \mathrm{~h}$ (T2), $12 \mathrm{~h}$ (T3) and $24 \mathrm{~h}$ (T4) after surgery. ${ }^{* *} \mathrm{P}<0.01 \mathrm{vs}$. the preoperative level in the sevoflurane group; ${ }^{\# \#} \mathrm{P}<0.01$ vs. the preoperative level in the propofol group. No significant difference was observed between the two groups at $\mathrm{T} 3$ and T4. Data are presented as the mean \pm standard deviation. cTnI, cardiac troponin I; NS, not significant.

statistical software (GraphPad Software, Inc., La Jolla, CA, USA) was used for data analysis.

\section{Results}

Patient characteristics. The basic characteristics of the patients in the two groups, including gender, age, BMI, NYHA level, the number of grafts and surgery duration, showed no significant differences between the two groups ( $P>0.05$; Table I). In addition, the dosages of sufentanil, vecuronium, nitroglycerin, esmolol, and phenylephrine used in surgery were not significantly different between the two groups $(\mathrm{P}>0.05)$. None of these patients required further surgery.

Hemodynamic and cardiac function measurements. Table II lists the preoperative and postoperative values of various hemodynamic and cardiac function measurements in the two groups. The results demonstrated no statistically significant differences in the preoperative hemodynamic variables, including HR, MAP, CVP, PAWP and PAMP, between the sevoflurane and propofol groups $(\mathrm{P}>0.05)$. By contrast, the $\mathrm{CO}$ and SV measured by pulmonary artery catheters showed a significant difference between the levels prior to surgery and subsequent to surgery in the sevoflurane group $(\mathrm{P}<0.05)$.

Cardiac marker expression. The expression of cTnI, CK-MB and LDH cardiac markers was determined using ELISA. As shown in Fig. 1, the protein expression of $\mathrm{cTnI}$ at $12 \mathrm{~h}$ after surgery was significantly increased in both groups, when compared with the corresponding preoperative values $(\mathrm{P}<0.01)$. The CK-MB levels were found to be significantly increased at $12 \mathrm{~h}$ after surgery in the propofol group only $(\mathrm{P}<0.01$; Fig. 2A), whereas significantly increased levels of LDH were observed at $12 \mathrm{~h}$ after surgery in both groups $(\mathrm{P}<0.01$; Fig. $2 \mathrm{~B})$. However, no significant differences were detected between the two groups in the expression of these variables (Figs. 1 and 2).

The expression levels of miR-499 and miR-208b were detected using RT-qPCR, and were found to be increased following surgery in both anesthesia groups, although the difference was only significant in the propofol group $(\mathrm{P}<0.05$; Fig. 3). There was no significant difference between the two
A
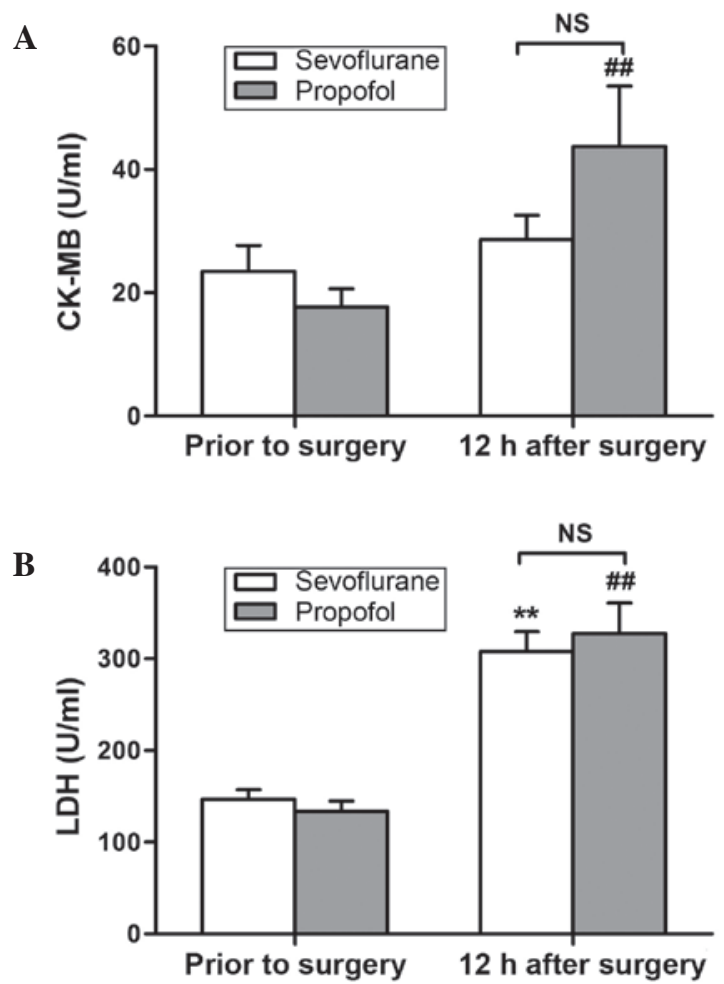

Figure 2. CK-MB and LDH expression levels prior to surgery and at $12 \mathrm{~h}$ after surgery. (A) CK-MB: ${ }^{\# \#} \mathrm{P}<0.01$ vs. prior to surgery. (B) $\mathrm{LDH}:{ }^{* *} \mathrm{P}<0.01$ vs. prior to surgery in the sevoflurane group; ${ }^{\# \#} \mathrm{P}<0.01$ vs. prior to surgery in the propofol group. No significant difference was observed between the two groups at $12 \mathrm{~h}$ after surgery. Data are presented as the mean \pm standard deviation. CK-MB, creatine kinase-MB; LDH, lactate dehydrogenase; NS, not significant.

groups prior to surgery ( $\mathrm{P}>0.05$; Fig. 3). However, at $12 \mathrm{~h}$ after surgery, the levels of the two miRNA markers were significantly reduced in the sevoflurane group when compared with the propofol group $(\mathrm{P}<0.05$; Fig. 3$)$.

\section{Discussion}

Previous studies have suggested that sevoflurane exerts a cardioprotective effect, mitigating myocardial damage, reducing the levels of CK-MB and cTnI, reducing the infarction area, and decreasing the incidence of arrhythmia and cardiac dysfunction (6-9). A recent study demonstrated that rat heart infarct size was significantly reduced in the sevoflurane preconditioned group compared with that in the ischemia/reperfusion group (24). The underlying mechanisms may include nuclear factor- $\kappa \mathrm{B}$ activation, upregulation of autophagy, and the attenuation of tumor necrosis factor- $\alpha$, interleukin-1 $\beta$ and caspase-3 expression (25-27). Another study found that sevoflurane preconditioning mediates against MIRI via caveolin-3-dependent cyclooxygenase-2 inhibition and antioxidative effects (28). In addition, sevoflurane postconditioning for $5 \mathrm{~min}$ was sufficient to activate protein kinase B and exert maximal cardioprotection against MIRI in isolated rat hearts (29). However, these studies used sevoflurane pre- or post-conditioning, along with propofol for induction and maintenance. Zaugg et al (30) found that propofol was able to cancel the cardioprotective effect of sevoflurane in a concentration-dependent manner. By contrast, 
A

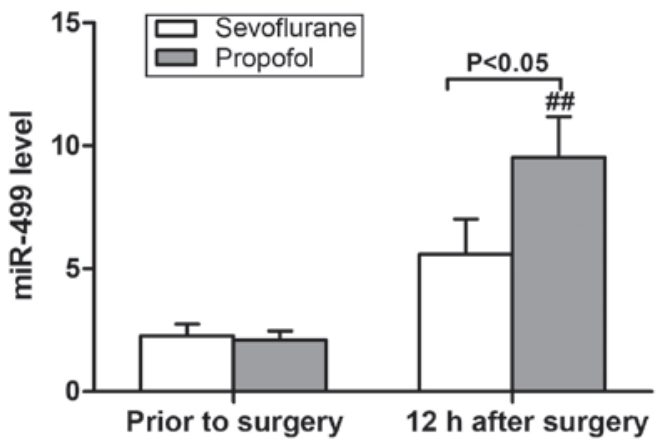

B

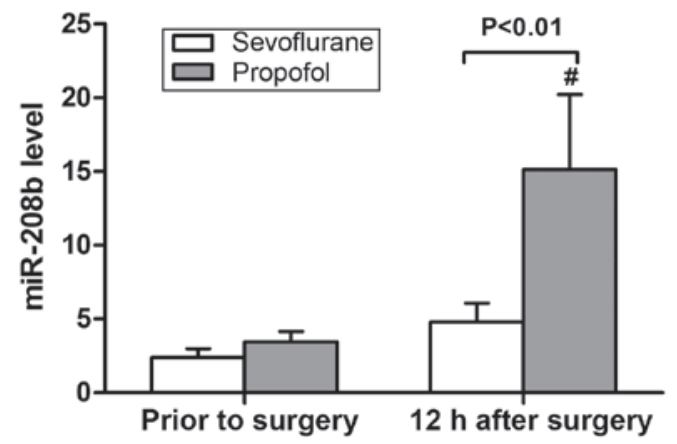

Figure 3. Relative expression levels of (A) miR-499 and (B) miR-208b. miR-499 and miR-208b were significantly increased from preoperative baseline to $12 \mathrm{~h}$ after surgery in the propofol group $\left({ }^{\# \#} \mathrm{P}<0.01\right.$ and $\left.{ }^{\#} \mathrm{P}<0.05\right)$. The level of miR-499 was significantly lower in the sevofluorane group when compared with the propofol group at $12 \mathrm{~h}$ after surgery $(\mathrm{P}<0.05)$. The level of miR-208b was also significantly lower in the sevoflurane group at $12 \mathrm{~h}$ after surgery $(\mathrm{P}<0.01)$. Data are presented as the mean \pm standard deviation.

certain other studies suggested the same protective effect for propofol $(31,32)$. Therefore, it is not certain which of the two drugs is mainly responsible for cardioprotection. The results of Jovic et al (33) indicated that sevoflurane and propofol led to cardiac protection via different mitochondrially-associated molecular mechanisms. It appears that sevoflurane acts by regulating cytochrome $c$ oxidase and ATP synthase, while the effects of propofol occur through regulation of cytochrome $c$, connexin 43, mtDNA transcription and uncoupling protein 2 (33). Other studies also showed the superiority of sevoflurane over propofol anesthesia. For instance, Ballester et al (34) compared the influence of sevoflurane and propofol on the levels of myocardial oxidative stress markers (F2-isoprostanes and nitrates/nitrites) in coronary sinus blood samples from patients undergoing OPCAB. Their data suggested that sevoflurane showed improved antioxidative properties compared with propofol (34).

In the present study, sevoflurane inhalation for VIMA without propofol was used to observe its clinical effect in comparison with propofol TIVA. Our data showed that there was no significant difference between the sevoflurane group and propofol group in hemodynamic variables and cardiac function, which is similar to the results of previous studies (35). The CO and SV of patients were higher subsequent to surgery in the sevoflurane group. Although the difference between the two group following surgery was not significant, sevoflurane may potentially benefit the clinical outcome.

Myocardial damage leads to the release of specific enzymes from myocardial cells into the blood. The most common enzymes include cTnI, CK-MB and LDH. cTnI has a high degree of myocardial specificity and sensitivity and is considered as a 'gold marker' of myocardial infarction (36). Numerous previous studies have investigated the influence of sevoflurane on myocardial enzyme levels in patients undergoing CABG, and the results have been contradictory (37-40). Law-Koune et al (37) and Hemmerling et al (38) found no difference between sevoflurane and propofol in their effects on $\mathrm{cTnI}$ and CK-MB in patients undergoing OPCAB $(37,38)$. However, two other studies showed the advantage of sevoflurane over propofol by reducing the levels of cardiac markers $(39,40)$. In the current study, the increase in the levels of cTnI following surgery was less in the sevoflurane group compared with the propofol group; however, the difference was not statistically significant. The influence of sevoflurane on $\mathrm{cTnI}$ may be associated with its inhaled concentration. Exposure to $2.8 \mathrm{vol} \%$ sevoflurane provides a better functional return and a lower percentage of infarction on reperfusion compared with $1.4 \mathrm{vol} \%$ sevoflurane (40). In the current study, the depth of anesthesia was adjusted by BIS (value, 40-50), so that only a low inhaled concentration of sevoflurane $(1.35 \pm 0.79$ vol\%) was required for the majority of time, with the exception of the induction period (8 vol\%). Piriou et al (41) also found that inhaled sevoflurane at 1MAC for $15 \mathrm{~min}$ had limited impact on cTnI. The CK-MB levels significantly increased in the sevoflurane and propofol groups following surgery. However, compared with the propofol group, CK-MB expression was significantly lower in the sevoflurane group after the surgery. Vikenes et al (42) identified that CK-MB was superior to the cardiac troponins in predicting long-term event-free survival subsequent to elective cardiac surgery in low-risk patients with stable symptoms undergoing $\mathrm{CAB}$ grafting. Furthermore, $\mathrm{LDH}$ is a nonspecific marker of myocardial injury, and LDH activity in the serum can be released from various types of damaged cells (43). In the present study, LDH expression increased following surgery in both groups; however, this was not significantly different between the two groups.

miRNAs are short non-coding RNAs, approximately 18-25 nucleotides long, which act as negative or positive regulators, primarily post-transcriptionally. Besides their intracellular function, recent studies have demonstrated that miRNAs can be exported or released by cells and circulate within the blood in a remarkably stable form $(44,45)$. The circulating miRNA patterns may be used as biomarkers for cardiovascular diseases. miR-499 and miR-208b are specifically expressed in myocardial tissue $(46,47)$. Recent animal experiments and clinical studies revealed that miR-499 and miR-208b increased subsequent to myocardial injury, and the degree of increase was positively correlated with the increase in cTnI levels $(48,49)$. Their diagnostic accuracy was found to be superior to other traditional biomarkers. Gidlof et al (50) collected plasma samples from 424 patients with suspected ACS treated in a coronary care unit, in order to measure cardio-enriched miRNAs. The authors found that miR-208b and miR-499 expression levels were higher in myocardial infarction patients, and established the association of increased miRNA levels with reduced systolic function subsequent to myocardial infarction and the risk of mortality or heart failure (50). The level of miR-499 in the plasma is very low in healthy individuals. Within $1-3 \mathrm{~h}$ after myocardial damage, this level begins to increase and reaches 
a peak at 3-12 h, and then decreases after 12-24 h (51). By contrast, miR-208b cannot be detected in healthy individuals, but appears in the plasma $1 \mathrm{~h}$ after myocardial injury, reaching a peak level at 3 and then decreasing after $12 \mathrm{~h}$ (46). A study by Ishikawa et al (52) showed that anesthetics cause numerous miRNA expression changes, and that the miRNA expression pattern was particular for each anesthetic, such as sevoflurane or propofol. Tanaka et al (53) found dynamic changes in miRNA expression caused by sevoflurane anesthesia on rats suffering from lung diseases.

The results of the present study showed that miR-499 and miR-208b were higher subsequent to surgery compared with those prior to surgery in both anesthesia groups. In addition, the two miRNA biomarkers were much lower in the sevoflurane group compared with the propofol group subsequent to surgery. This confirmed that the cardioprotective effect of sevoflurane on patients undergoing OPCAB is superior to that of propofol. Furthermore, the current study established an association between sevoflurane and miRNAs in patients undergoing OPCAB for the first time. The small number of patients included in the study limits our conclusion, and thus larger numbers should be used to further assess the cardioprotective effect of sevoflurane. Whether the results can be extended to other cardiac patients requires further research.

In conclusion, the present study demonstrated that VIMA with sevoflurane in patients undergoing OPCAB resulted in improved cardiac protection compared with propofol TIVA, and this effect was indicated based on the levels of two sensitive biomarkers, circulating miR-499 and miR-208b.

\section{References}

1. Parissis H, Mbarushimana S, Ramesh BC, Parissis $M$ Lampridis S, Mhandu $\mathrm{P}$ and Al-Alao B: The impact of off-pump surgery in end-organ function: Practical end-points. J Cardiothorac Surg 10: 158, 2015.

2. Gonenc A, Hacişevki A, Griffiths HR, Torun M, Bakkaloglu B and Simsek B: Free radical reaction products and antioxidant capacity in beating coronary artery surgery compared to conventional bypass. Biochemistry (Mosc) 76: 677-685, 2011

3. Leslie JB: Incidence and aetiology of perioperative hypertension. Acta Anaesthesiol Scand Suppl 99: 5-9, 1993.

4. Yao YT, Fang NX, Shi CX and Li LH: Sevoflurane postconditioning protects isolated rat hearts against ischemia-reperfusion injury. Chin Med J (Engl) 123: 1320-1328, 2010.

5. Coetzee JF, Roux PJ, Genade S and Lonchner A: Reduction of postischemic contractile dysfunction of the isolated rat heart by sevoflurane: Comparison with halothane. Anesth Analg 90: 1089-1097, 2000.

6. De Hert SG, Broecke PW, Mertens E Van Sommeren EW, De Blier IG, Stockman BA and Rodrigus IE: Sevoflurane but not propofol preserves myocardial function in coronary surgery patients. Anesthesiology 97: 42-49, 2002.

7. De Hert SG, Van der Linden PJ, Cromheecke S, Meeus R Nelis A, Van Reeth V, ten Broecke PW, De Blier IG, Stockman BA and Rodrigus IE: Cardioprotective properties of sevoflurane in patients undergoing coronary surgery with cardiopulmonary bypass are related to the modalities of its administration. Anesthesiology 101: 299-310, 2004.

8. Haroun-Bizri S, Khoury SS, Chehab IR, Kassas CM and Baraka A: Does isoflurane optimize myocardial protection during cardiopulmonary bypass? J Cardiothorac Vasc Anesth 15: 418-421, 2001.

9. JulierK,daSilvaR,GarciaC,BestmannL,FrascaroloP,Zollinger A, Chassot PG, Schmid ER, Turina MI, von Segesser LK, et al: Preconditioning by sevoflurane decreases biochemical markers for myocardial and renal dysfunction in coronary artery bypass graft surgery: A double-blinded, placebo-controlled, multicenter study. Anesthesiology 98: 1315-1327, 2003.
10. Fisher LA, Beckman JA, Brown KA, Calkins H, Chaikof E, Fleischmann KE, Freeman WK, Froehlich JB, Kasper EK, Kersten JR, et al: ACC/AHA 2007 guidelines on perioperative cardiovascular evaluation and care for noncardiac surgery: A report of the American college of cardiology/American heart association task force on practice guidelines. Circulation 116: e418-e499, 2007.

11. Landoni G, Bignami E, Oliviero F and Zangrillo A: Halogenated anaedthetics and cardiac protection in cardiac and non-cardiac anesthesia. Ann Card Anesth 12: 4-9, 2009.

12. Landoni G, Biondi-Zoccai GG, Zangrillo A, Bignami E, D'Avolio S, Marchetti C, Calabrò MG, Fochi O, Guarracino F, Tritapepe L, et al: Desflurane and sevoflurane in cardiac surgery: A meta-analysis of randomized clinical trials. J Cardiothorac Vasc Anesth 21: 502-511, 2007.

13. Hamm CW, Goldmann BU, Heeschen C, Kreymann G, Berger $\mathrm{J}$ and Meinertz T: Emergency room triage of patients with acute chest based on rapid testing for troponin $\mathrm{T}$ or troponin I. N Engl J Med 337: 1648-1653, 1997.

14. Davies E, Gawad Y, Takahashi M, Shi Q, Lam P, Styba G, Lau A, Heeschen C, Usategui M and Jackowski G: Analytical performance and clinical utility of a sensitive immunoassay for determination of human cardiac troponin I. Clin Biochem 30: 479-490, 1997

15. Lewandrowski K, Chen A and Januzzi J: Cardiac markers for myocardial infarction. Am J Clin Pathol 118 (Suppl): S93-S99, 2002.

16. Dekker MS, Mosterd A, van't Hof AW and Hoes AW: Novel biochemical markers in suspected acute coronary syndrome: Systematic review and critical appraisal. Heart 96: 1001-1010, 2010.

17. Lewis BP, Shih IH, Jones-Rhoades MW, Bartel DP and Burge CB: Prediction of mammalian microRNA targets. Cell 115: 787-798, 2003.

18. Sluijter JP, van Mil A, van Vliet P, Metz CH, Liu J, Doevendans PA and Goumans MJ: MicroRNA-1 and -499 regulate differentiation and proliferation in human-derived cardiomyocyte progenitor cells. Arterioscler Thromb Vasc Biol 30: 859-868, 2010.

19. van Rooij E, Sutherland LB, Liu N, Williams AH, McAnally J, Gerard RD, Richardson JA and Olson EN: A signature pattern of stress-responsive microRNAs that can evoke cardiac hypertrophy and heart failure. Proc Natl Acad Sci USA 103: 18255-18260, 2006.

20. Oerlemans MI, Mosterd A, Dekker MS, de Vrey EA, van Mil A, Pasterkamp G, Doevendans PA, Hoes AW and Sluijter JP: Early assessment of acute coronary syndromes in the emergency department: The potential diagnostic value of circulating microRNAs. EMBO Mol Med 4: 1176-1185, 2012.

21. Devaux Y, Vausort M, Goretti E, Nazarov PV, Azuaje F, Gilson G, Corsten MF, Schroen B, Lair ML and Heymans S: Use of circulating microRNAs to diagnose acute myocardial infarction. Clin Chem 58: 559-567, 2012.

22. Lequin RM: Enzyme immunoassay (EIA)/enzyme-linked immunosorbent assay (ELISA). Clin Chem 51: 2415-2418, 2005.

23. Livak KJ and Schmittgen TD: Analysis of relative gene expression data using real-time quantitative PCR and the 2(-Delta Delta C(T)) Method. Methods 25: 402-408, 2001.

24. Qiao S, Xie H, Wang C, Wu X, Liu H and Liu C: Delayed anesthetic preconditioning protects against myocardial infarction via activation of nuclear factor- $\kappa \mathrm{B}$ and upregulation of autophagy. J Anesth 27: 251-260, 2013.

25. Torina AG, Reichert K, Lima F, de Souza Vilarinho KA, de Oliveira PP, do Carmo HR, de Carvalho DD, Saad MJ, Sposito AC and Petrucci O: Diacerein improves left ventricular remodeling and cardiac function by reducing the inflammatory response after myocardial infarction. PLoS One 10: e0121842, 2015.

26. Wu X, He L, Chen F, He X, Cai Y, Zhang G, Yi Q, He M and Luo J: Impaired autophagy contributes to adverse cardiac remodeling in acute myocardial infarction. PLoS One 9: e112891, 2014.

27. Liu Q: Lentivirus mediated interference of Caspase-3 expression ameliorates the heart function on rats with acute myocardial infarction. Eur Rev Med Pharmacol Sci 18: 1852-1858, 2014.

28. Zhao J, Wang F, Zhang Y, Jiao L, Lau WB, Wang L, Liu B, Gao E, Koch WJ and Ma XL: Sevoflurane preconditioning attenuates myocardial ischemia/reperfusion injury via caveolin-3-dependent cyclooxygenase-2 inhibition. Circulation 128 (11 Suppl 1): S121-S129, 2013.

29. Yao YY, Zhu MH, Zhang FJ, Wen CY, Ma LL, Wang WN, Wang CC, Liu XB, Yu LN, Qian LB, et al: Activation of Akt and cardioprotection against reperfusion injury are maximal with only five minutes of sevoflurane post-conditioning in isolated rat hearts. J Zhejiang Univ Sci B 14: 511-517, 2013. 
30. Zaugg M, Wang L, Zhang L, Lou PH, Lucchinetti E and Clanachan AS: Choice of anesthetic combination determines Ca2+ leak after ischemia-reperfusion injury in the working rat heart: Favorable versus adverse combinations. Anesthesiology 116: 648-657, 2012.

31. Samir A, Gandreti N, Madhere M, Khan A, Brown M and Loomba V: Anti-inflammatory effects of propofol during cardiopulmonary bypass: A pilot study. Ann Card Anaesth 18: 495-501, 2015.

32. Xia WF, Liu Y, Zhou QS, Tang QZ and Zou HD: Protective effect of propofol and its relation to postoperation recovery in children undergoing cardiac surgery with cardiopulmonary bypass Pediatr Cardiol 32: 940-946, 2011.

33. Jovic M, Stancic A, Nenadic D, Cekic O, Nezic D, Milojevic P, Micovic S, Buzadzic B, Korac A, Otasevic V, et al: Mitochondrial molecular basis of sevoflurane and propofol cardioprotection in patients undergoing aortic valve replacement with cardiopulmonary bypass. Cell Physiol Biochem 29: 131-142, 2012.

34. Ballester M, Llorens J, Garcia-de-la-Asuncion J, Perez-Griera J, Tebar E, Martinez-Leon J, Belda J and Juez M: Myocardial oxidative stress protection by sevoflurane vs. propofol: A randomised controlled study in patients undergoing off-pump coronary artery bypass graft surgery. Eur J Anaesthesiol 28: 874-881,2011.

35. El Azab SR, Scheffer GJ, Rosseel PM and De Lange JJ: Induction and maintenance of anaesthesia with sevoflurane in comparison to high dose opioid during coronary artery bypass surgery. Eur J Anaesthesiol 17: 336-338, 2000.

36. Futterman LG and Lemberg L: SGOT, LDH, HBD, CPK, CK-MB, MB1MB2, cTnT, cTnC, cTnI. Am J Crit Care 6: 333-338, 1997.

37. Law-Koune JD, Raynaud C, Liu N, Dubois C, Romano M and Fischler M: Sevoflurane-remifentanyl versus propofol-remifentanyl anesthesia at a similar bispectral level for off-pump coronary artery surgery: No evidence of reduced myocardial ischemia. J Cardiothorac Vasc Anesth 20: 484-492, 2006.

38. Hemmerling T, Olivier J, Le N, Prieto I and Bracco D: Myocardial protection by isoflurane vs. sevoflurane in ultra-fast-track anaesthesia for off-pump aortocoronary bypass grafting. Eur J Anaesthesiol 25: 230-236, 2008.

39. Conzen PF, Fischer S, Detter C and Peter K: Sevoflurane provides greater protection of the myocardium than propofol in patients undergoing off-pump coronary artery bypass surgery. Anesthesiology 99: 826-833, 2003.

40. Riess ML, Kevin LG, Camara AK, Heisner JS and Stowe DF: Dual exposure to sevoflurane improves anesthetic preconditioning in intact hearts. Anesthesiology 100: 569-574, 2004.

41. Piriou V, Mantz J, Goldfarb G, Kitakaze M, Chiari P, Paquin S, Cornu C, Lecharny JB, Aussage P, Vicaut E, et al: Sevoflurane preconditioning at $1 \mathrm{MAC}$ only provides limited protection in patients undergoing coronary artery bypass surgery: A randomized bi-centre trial. Br J Anaesth 99: 624-631, 2007.
42. Vikenes K, Andersen KS, Melberg T,Farstad Mand Nordrehaug JE: Long-term prognostic value of cardiac troponin I and T versus creatine kinase-MB mass after cardiac surgery in low-risk patients with stable symptoms. Am J Cardiol 106: 780-786, 2010.

43. Ekaney ML, Otto GP, Sossdorf M, Sponholz C, Boehringer M, Loesche W, Rittirsch D, Wilharm A, Kurzai O, Bauer M and Claus RA: Impact of plasma histones in human sepsis and their contribution to cellular injury and inflammation. Crit Care 18: 543 , 2014.

44. De Rosa S and Indolfi C: Circulating microRNAs as Biomarkers in Cardiovascular Diseases. EXS 106: 139-149, 2015.

45. Schulte C, Molz S, Appelbaum S, Karakas M, Ojeda F, Lau DM, Hartmann T, Lackner KJ, Westermann D, Schnabel RB, et al: miRNA-197 and miRNA-223 Predict Cardiovascular Death in a Cohort of Patients with Symptomatic Coronary Artery Disease. PLoS One 10: e0145930, 2015.

46. Ji X, Takahashi R, Hiura Y, Hirokawa G, Fukushima Y and Iwai N: Plasma miR-208 as a biomarker of myocardial injury. Clin Chem 55: 1944-1949, 2009.

47. Reddy A, Zheng Y, Jagadeeswaran G, Macmil SL Graham WB, Roe BA, Desilva U, Zhang W and Sunkar R: Cloning, characterization and expression analysis of porcine microRNAs. BMC genomics 10: 65, 2009

48. Gidlöf O, Andersson P, van der Pals J, Götberg M and Erlinge D: Cardiospecific microRNA plasa levels correlate with troponin and cardiac function in patients with ST elevation myocardial infarction, are selectively dependent on renal elimination and can be detected in urine samples. Cardiology 118: 217-226, 2011.

49. Olivieri F, Antonicelli R, Lorenzi M, D'Alessandra Y, Lazzarini R, Santini G, Spazzafumo L, Lisa R, La Sala L, Galeazzi R, et al: Diagnostic potential of circulating miR-499-5p in elderly patients with acute non ST-elevation myocardial infarction. Int J Cardiol 167: 531-536, 2013.

50. Gidlof O, Smith JG, Miyazu K, Gilje P, Spencer A, Blomquist S and Erlinge D: Circulating cardio-enriched microRNAs are associated with long-term prognosis following myocardial infarction. BMC Cardiovasc Disord 13: 12, 2013.

51. Corsten MF, Dennert R, Jochems S, Kuznetsova T, Devaux Y, Hofstra L, Wagner DR, Staessen JA, Heymans S and Schroen B: Circulating MicroRNA-208b and MicroRNA-499 reflect myocardial damage in cardiovascular disease. Circ Cardiovasc Genet 3: 499-506, 2010 .

52. Ishikawa M, Tanaka S, Arai M, Genda Y and Sakamoto A Differences in microRNA changes of healthy rat liver between sevoflurane and propofol anesthesia. Anesthesiology 117: 1245-1252, 2012.

53. Tanaka S, Ishikawa M, Arai M, Genda Y and Sakamoto A: Changes in microRNA expression in rat lungs caused by sevoflurane anesthesia: A TaqMan ${ }^{\circledR}$ low-density array study. Biomed Res 33: 255-263, 2012. 